Open Access

Zlatko Matjačić*, Matjaž Zadravec and Andrej Olenšek

\title{
Comparison of dynamic balancing responses following outward lateral perturbations during walking of healthy and post-stroke subjects
}

DOI 10.1515/cdbme-2017-0003

\begin{abstract}
Efficient dynamic balancing and movement coordination during walking are essential for stability. The objective of this preliminary study was to assess dynamic balancing responses in a selected post-stroke subject and to compare them with those assessed in neurologically intact individual. Balance Assessment Robot, a haptic robot that interfaces to a pelvis of a subject walking on an instrumented treadmill, was used to deliver perturbing pushes to the pelvis. We have assessed centre-of-pressure (CoP) and horizontal components of ground reaction forces (GRF) following outward pushes. The results have shown that depending on the amplitude of a perturbing push neurologically intact individual responded predominantly by "ankle" and "hip" strategies at lower amplitude of perturbation and "ankle" and "stepping" strategies at higher amplitude of perturbation. Post-stroke subject responded mainly by "ankle" and "hip" strategies when perturbed on the sound leg while the response when perturbed on the impaired leg was similar to the one observed in healthy subject. These preliminary results indicate that post-stroke subjects might be reluctant or not able to perform "cross step" with their impaired leg which is needed when counteracting outward perturbation.
\end{abstract}

Keywords: ankle strategy; hip strategy; perturbed walking; stepping response.

\section{Introduction}

Dynamic balancing is immanently imbedded in human walking. Even if we consider steady walking in the absence

\footnotetext{
*Corresponding author: Zlatko Matjačić, University rehabilitation institute Republic of Slovenia Linhartova 51, SI-1000 Ljubljana, Slovenia, E-mail: zlatko.matjacic@ir-rs.si

Matjaž Zadravec and Andrej Olenšek: University rehabilitation institute Republic of Slovenia Linhartova 51, SI-1000 Ljubljana, Slovenia, E-mail: matjaz.zadravec@ir-rs.si (M. Zadravec); andrej.olensek@ir-rs.si (A. Olenšek)
}

of perturbations appropriate relationship between centre of pressure (COP) and centre of mass ( $\mathrm{COM})$, which is the main mechanism of balance control, needs to be maintained from step to step [1]. Additionally, during walking we may experience perturbations in a form of slip, trip or we may even bump into things or people, which challenges stability [2]. Poor balance particularly in the frontal plane has been suggested as a major reason for falls in elderly and neurologically impaired population [3].

Brain stroke in terms of movement results in hemiparesis that affects muscle strength and coordination primarily on the impaired side of the body. Consequently, also dynamic balancing abilities in post-stroke population are impaired depending on each individual case. There is a need to develop fall-safe techniques that will enable objective assessment of balancing abilities constituting a base for development of suitable patient-specific activities that evoke balancing responses within training of walking in post-stroke population. We have recently developed innovative admittance-controlled balance assessment robot (BAR) which can be placed either on mobile platform enabling over ground walking or on an instrumented treadmill (BAR-TM) and allows assessment of COP, COM and ground reaction force (GRF) in all three components following perturbing pushes [4].

The objective of this contribution was to assess balancing responses in a selected post-stroke subject and compare them to balancing responses obtained in a height- and weight-matched healthy individual. The specific aim was to explore differences in balancing responses resulting from exactly the same pushes applied to the pelvis in the outward, lateral direction, which is the most demanding perturbation to be counteracted during walking as it requires cross-stepping.

\section{Methods}

We have recently developed a robot that interfaces to the pelvis of a walking subject and enables unhindered 


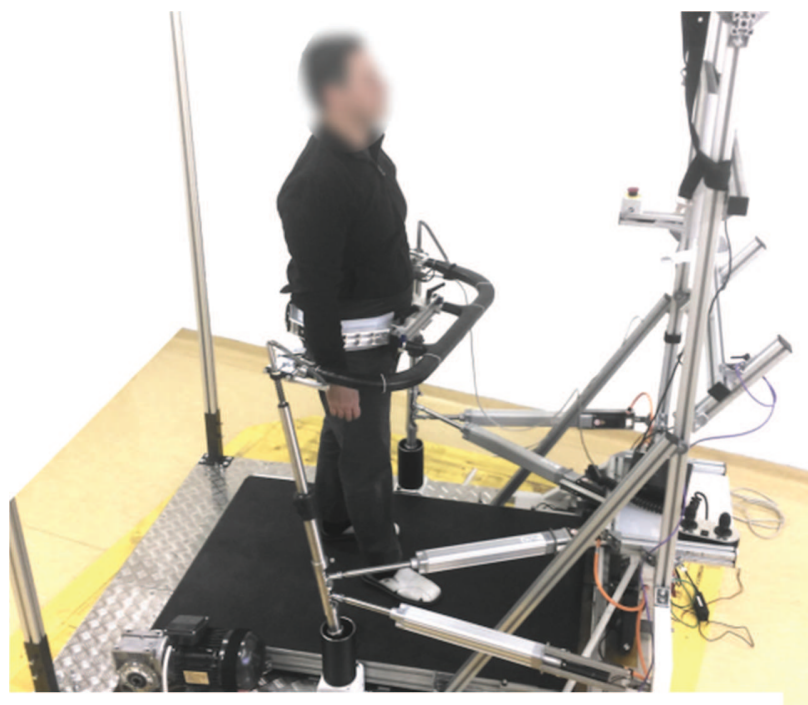

Figure 1: Healthy subject walking on BAR-TM.
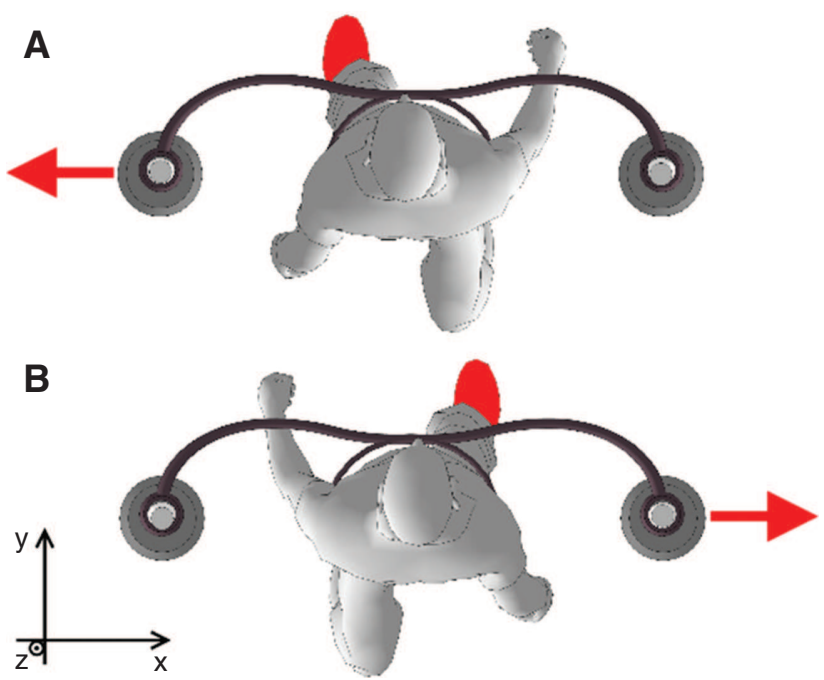

Figure 2: Perturbation timing (left - A, right - B heel strike), outward perturbation directions and coordinate system.

movement of the pelvis in all six degrees of freedom (DOF) as shown in Figure 1. Three DOFs (translation of pelvis in the vertical direction and rotation of pelvis around the sagittal and lateral axis) are passive and spring loaded while the remaining three DOFs (translation of pelvis in sagittal and lateral directions and rotation around the vertical axis) are actuated and admittance-controlled. The robot is placed around an instrumented treadmill (BARTM) and is capable of delivering highly repeatable and well-defined perturbations in the directions indicated in Figure 2. Detailed description on the mechanism, control and performance of BAR-TM is given in [4].
A post-stroke subject (age 55 years, height $179 \mathrm{~cm}$, weight $110 \mathrm{~kg}) 18$ months after stroke and a healthy subject (age 33 years, height $178 \mathrm{~kg}$, weight $108 \mathrm{~kg}$ ) participated in this study. Both subjects were walking on BAR-TM system at speed of $0.5 \mathrm{~m} / \mathrm{s}$ during unperturbed experimental condition to obtain baseline measurement of COP, COM and GRF in lateral (x axis) and sagittal (y axis) directions. Post-stroke subject wore an ankle-foot orthosis that stabilised ankle in the medio-lateral direction. Subsequently balancing responses to outward directed perturbations (perturbing forces were $80 \mathrm{~N}$ and $120 \mathrm{~N}$ for healthy subject and $120 \mathrm{~N}$ for post-stroke subject; duration of force impulse was 150 $\mathrm{ms}$ ) at the same walking speed were assessed. These parameters were based on experience gained in our previous study [4]. Perturbation commenced at left heel strike in healthy subject while in post stroke subject we collected COP, COM and GRF measurements following outward perturbations at heel strike of unimpaired left leg (Figure 2A) and at heel strike of impaired right leg (Figure 2B). Half gait cycle prior to and two and a half cycle after perturbation commencement were analysed where mean values and standard deviations of five repeated trials were calculated.

\section{Results}

Figure 3 shows a complete set of measurements in a healthy subject for unperturbed and perturbed (amplitude $120 \mathrm{~N}$ ) walking. During the perturbation period interaction force $\mathrm{F}_{\mathrm{X}}$ between the robot and the walking subject dis-placed pelvis laterally, later in the first gait cycle pronounced pelvis rotation was observed while the movement of pelvis in sagittal plane did not differ much between both experimental conditions. Comparing $\mathrm{COP}_{\mathrm{y}}$ and $\mathrm{COM}_{\mathrm{y}}$ does not show substantial differences; however, substantial differences can be seen in lateral direction. $\mathrm{COP}_{\mathrm{x}}$ in perturbed condition is displaced laterally during left stance very soon after perturbation commencement (at around 10\% of gait cycle) - this represents so called "ankle strategy" [1]; in subsequent two steps COP is displaced further laterally due to placement of next right and left steps this represents so called "stepping strategy"; observing $\mathrm{GRF}_{\mathrm{x}}$ shows a small but noticeable peak immediately after perturbation commencement - this represents so called "hip strategy" [1]. It can be noticed that "hip strategy" was just indicated but it was very soon dismissed in order to decrease acceleration of $\mathrm{COM}_{\mathrm{x}}$ toward medial direction (from around 20\%-50\%). This is consistent as with such response the outward movement of $\mathrm{COM}_{\mathrm{X}}$ is not opposed but subject rather yielded to the action of perturbation. This response was maintained also during the next, right step 

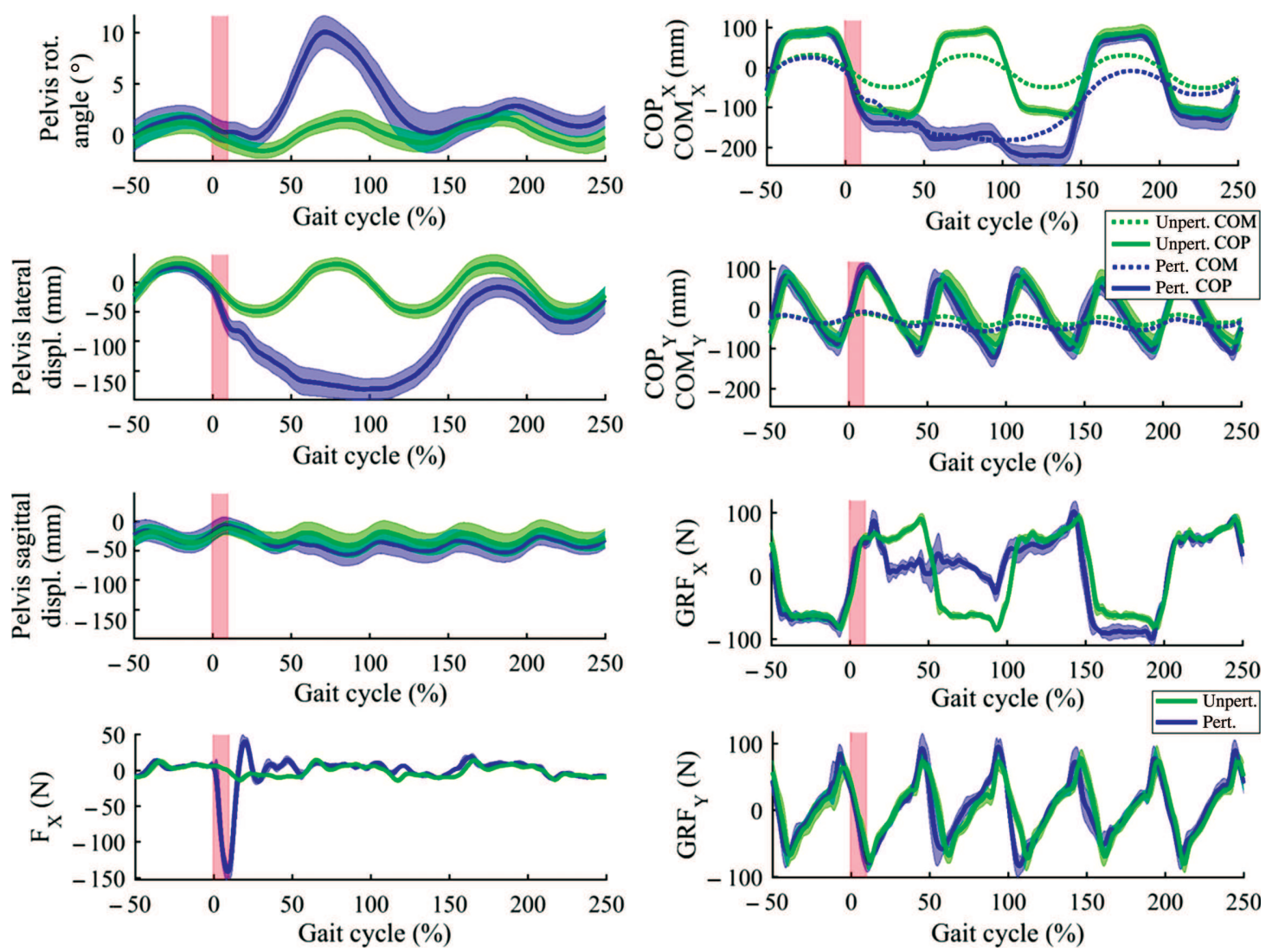

Figure 3: Pelvis kinematics, perturbation force action on the pelvis, COP, COM and GRF trajectories as assessed in the healthy subject walking at speed of $0.5 \mathrm{~m} / \mathrm{s}$ following perturbation force impulse of $120 \mathrm{~N}(150 \mathrm{~ms})$.

(from 50\%-100\%). Thus, the two dominant mechanisms used in responding to this perturbation were "ankle and stepping strategies" [1].

Figures 4 and 5 show unperturbed and perturbed $\mathrm{COP}_{\mathrm{x}}$, $\mathrm{COM}_{\mathrm{x}}$ and $\mathrm{GRF}_{\mathrm{x}}$ trajectories assessed at different amplitudes of perturbing forces in healthy subject. When the perturbing force was lower $(80 \mathrm{~N})$ it can be noticed that the subject predominantly responded by "ankle strategy" (shift of $\mathrm{COP}_{\mathrm{x}}$ more laterally from $10 \%-50 \%$ of gait cycle) and "hip strategy" (burst in $\mathrm{GRF}_{\mathrm{X}}$ from $10 \%-20 \%$ of gait cycle). "Stepping strategy" as seen by slight shift of $\mathrm{COP}_{\mathrm{x}}$ more medially in the next, right step was utilized to much smaller extent. However, when perturbing force was higher (120 N) the predominant strategies were "ankle" and "stepping" as already described in more detail above when describing Figure 3.

Figures 6 and 7 show unperturbed and perturbed $\mathrm{COP}_{\mathrm{x}}, \mathrm{COM}_{\mathrm{x}}$ and $\mathrm{GRF}_{\mathrm{x}}$ trajectories assessed at the same amplitudes of perturbing forces $(120 \mathrm{~N})$ but at different time instants of perturbation commencement in post-stroke subject. When the perturbation commenced at heel strike on the left, unimpaired leg the predominant approach of the post-stroke subject was to respond by "hip strategy"
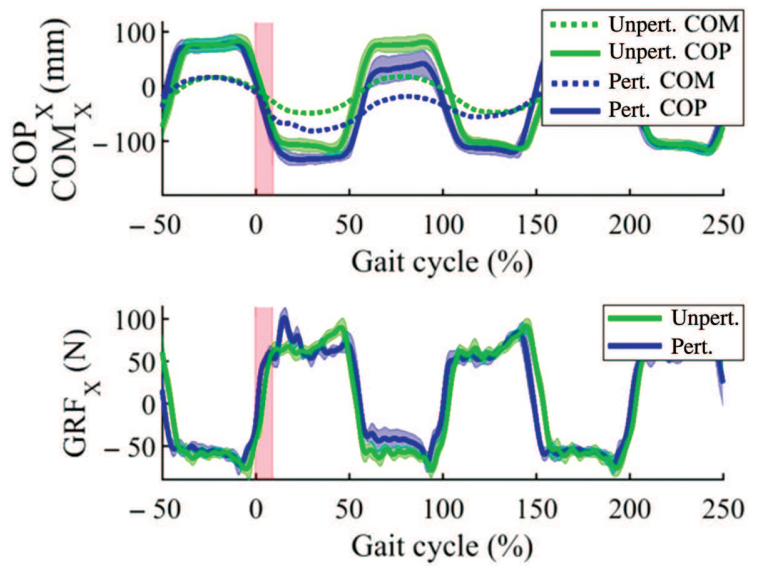

Figure 4: Healthy subject $(80 \mathrm{~N})$.

(significant burst in $\mathrm{GRF}_{\mathrm{x}}$ from $10 \%-30 \%$ of gait cycle); there is absence of "ankle strategy" while "stepping strategy" is just indicated (from $50 \%-100 \%$ of gait cycle). When the perturbation commenced at heel strike on the right, impaired leg the predominant approach of the post-stroke subject was "ankle strategy" (lateral shift of $\mathrm{COP}_{\mathrm{x}}$ from $10 \%-50 \%$ of gait cycle) and "stepping strategy" in the next two steps. 

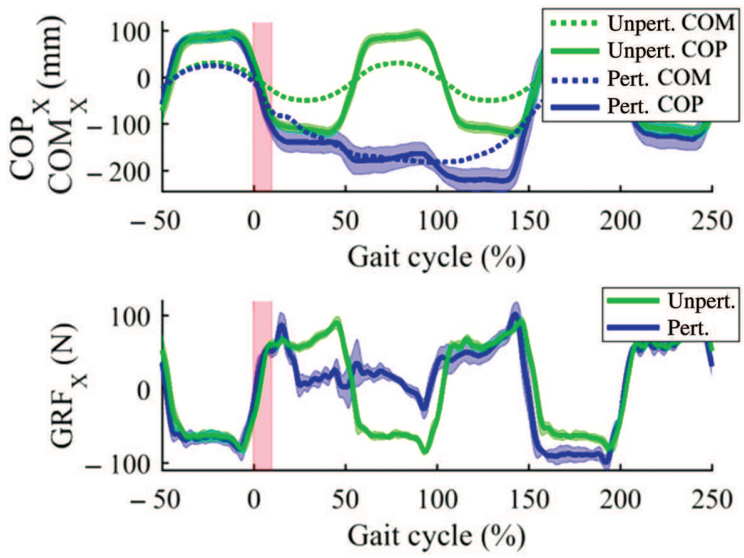

Figure 5: Healthy subject (120 N).
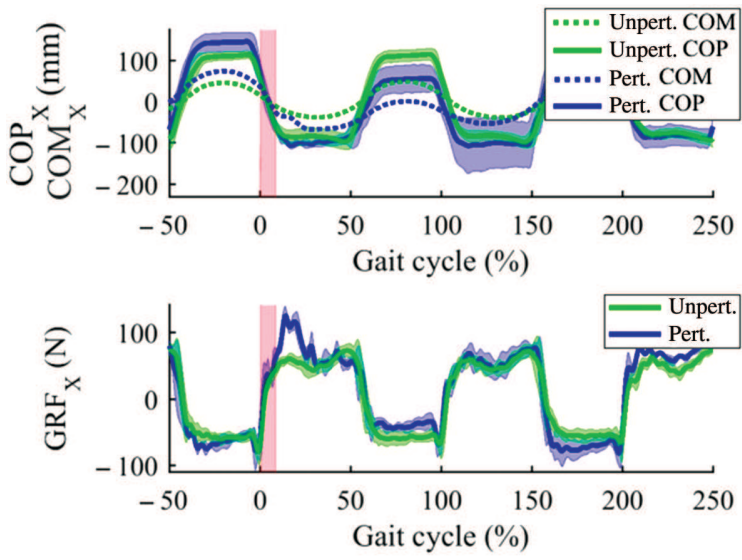

Figure 6: Post stroke subject - unimpaired side (120 N).

\section{Discussion}

In this preliminary study we have explored balancing responses following perturbations in the outward, lateral directions in healthy and post-stroke subjects. The results have shown that there is a clear difference in performance of the post-stroke subject depending on whether the perturbation kicked-in when entering stance phase at unimpaired or impaired side. There was substantial change in the strategies used confirming previous observations that crossstepping with impaired limb which is required to execute "stepping strategy" represents a significant challenge to post-stroke subject. The results form healthy subject has shown that the intensity of perturbation plays a significant role in selection of suitable set of responses. It seems that all three strategies ("ankle", "hip" and "stepping") are always used in shaping balancing response but the exact share of each depends on the various perturbation parameters (in our case the amplitude of the push).

In our further work we plan to extend the study on more subjects and to assess also electromyographic signals from the relevant lower limb muscles to further elaborate
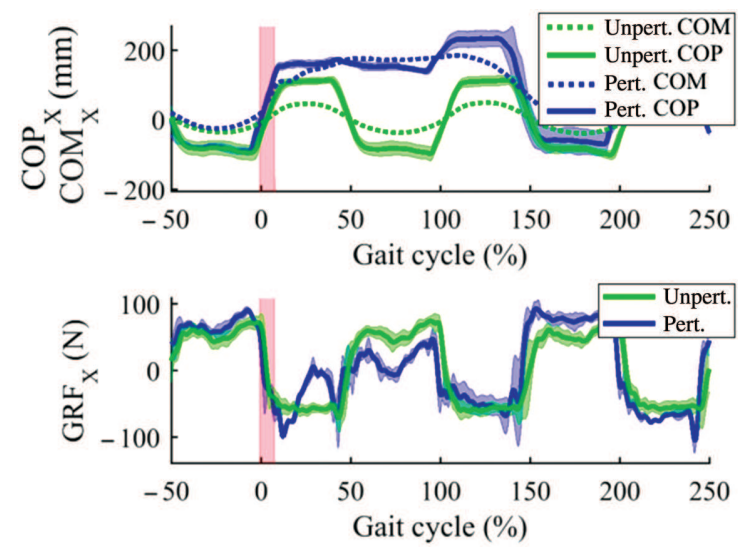

Figure 7: Post stroke subject - impaired side (120 N).

the role and hierarchical dependency of each strategy used in particular balancing response. In terms of possible training of balancing responses in post-stroke subjects our results indicate that the assessment as performed in this study would be valuable in determining suitable subjectspecific perturbation parameters to be used subsequently in training in each individual post-stroke subject.

\section{Author's Statement}

Research funding: This research was partially supported by the Slovenian Research Agency under research project L2-5471, research program number P2-0228 and by the European Commission $7^{\text {th }}$ Framework Programme for research, technological development and demonstration as part of the BALANCE project under grant agreement number 601003. Conflict of interest: Authors state no conflict of interest. Informed consent: Informed consent has been obtained from all individuals included in this study. Ethical approval: The research related to human use complies with all the relevant national regulations, institutional policies and was performed in accordance with the tenets of the Helsinki Declaration, and has been approved by the authors' institutional review board.

\section{References}

[1] Hof AL. The equations of motion for a standing human reveal three mechanisms for balance. J Biomech. 2007;40:451-7.

[2] Maki BE, Mcllroy WE. Control of rapid limb movements for balance recovery: age-related changes and implications for fall prevention. Age Ageing 2006;35(Suppl 2):ii12-8.

[3] Wang TY, Bhatt T, Yang F, Pai YC. Adaptive control reduces tripinduced forward gait instability among young adults. J Biomech. 2012;45:1169-75.

[4] Olenšek A, Zadravec M, Matjaéiæ Z. A novel robot for imposing perturbations during overground walking: mechanism, control and normative stepping responses. J Neuroeng Rehabil. 2016;13:55. 